Dulmen, S. van, Smits, L., Eide, H. Filling in memory gaps through emotional communication: promising pathways in caring for persons with dementia. Patient Education and Counseling: 2017, 100(11), 2121-2124

\begin{tabular}{|l|l|}
$\begin{array}{l}\text { Postprint } \\
\text { Version }\end{array}$ & 1.0 \\
\hline Journal website & http://www.pec-journal.com/article/S0738-3991(17)30360-9/fulltext \\
\hline Pubmed link & $\underline{\text { https://www.ncbi.nlm.nih.gov/pubmed/28641992 }}$ \\
\hline DOI & $10.1016 /$ j.pec.2017.06.014 \\
\hline
\end{tabular}

This is a NIVEL certified Post Print, more info at http://www.nivel.eu

\title{
Filling in memory gaps through emotional communication; promising pathways in caring for persons with dementia
}

\author{
SANDRA VAN DULMEN ${ }^{\mathrm{A}, \mathrm{B}, \mathrm{C},}$, LIES SMITS $^{\mathrm{D}}$, HILDE EIDE $^{\mathrm{C}}$ \\ a NIVEL (Netherlands institute for health services research), Utrecht, The Netherlands \\ b Department of Primary and Community Care, Radboud University Medical Center, \\ Nijmegen, The Netherlands \\ c Science Centre Health and Technology, Faculty of Health Sciences, University College of \\ Southeast Norway, Drammen, Norway \\ d Canisius Wilhelmina Hospital, Nijmegen, The Netherlands
}

\begin{abstract}
Objective

To explore in what way emotional communication can enhance the memory of people with (different types of) dementia.

Methods

Relevant studies published after 2000 were searched using the terms: dementia, positive, words, communication, recall, and memory. Papers were included that reported results of studies with people with dementia that investigated memory effects of communication with either an emotionally valent content or context.

Results

Twelve papers grouped under four prevailing themes (pictures, facial emotions, stories and words) are described. The studies provide mixed results: in some studies negative emotional information enhances memory in older people with dementia, in other studies positive emotional information is helpful or hardly any effect is found.

Conclusion

Emotional communication seems to enhance memory in people with dementia. None of the studies described focused on the association between personally relevant, emotionally valent information and memory, so further research is needed.

Practice implications

Caregivers in dementia care should realize that 1) the information they provide might carry an emotional valence, and 2) this valence might influence the extent to which people with dementia remember information.
\end{abstract}


Dulmen, S. van, Smits, L., Eide, H. Filling in memory gaps through emotional communication: promising pathways in caring for persons with dementia. Patient Education and Counseling: 2017, 100(11), 2121-2124

\section{INTRODUCTION}

"My mother forgets almost everything. We need to tell her ten times a day that we already had lunch and listen to the same story about how she met my father 60 years ago over and over again. We just respond as if we never heard this before, which is okay. But, when I tell her just before running errands, that her oldest granddaughter has found her dream apartment, which she is very pleased to hear, she comes back to this news upon my return one hour later by saying: 'Really, I am so glad that she found a new apartment'! I have noticed this before, information with a positive emotional valence seems to be remembered better." [Daughter of 85-year old woman with dementia] If information is indeed remembered better or longer when it carries a positive emotional valence, this power of emotions might be used in a purposeful way to (temporarily) fill in gaps in the memory of older people with dementia. Apart from this direct therapeutic value, this could also lighten the burden of care for formal and informal caregivers. After all, as people with dementia increasingly struggle with initiating and performing daily activities as their condition deteriorates, at a given moment, their contact with caregivers is all that remains. Putting effort into increasing the quality and output of these contacts is therefore warranted. It is an established fact that stimuli that are connected to emotions will be better remembered than non emotional or neutral stimuli [1]. An important question is whether this is also the case for persons with dementia. If so, this may have clinical implications. A second question is if a possible recall effect is dependent on the severity of the dementia.

Much research into the role of emotions in caring for people with dementia has focused on (autobiographical) memory for events and people that happened in the past; old pictures and narratives but also smells and music have shown to be comforting for people. This insight has successfully been applied to diminish disturbing and withdrawn behavior as part of multisensory stimulation ('snoezelen') integrated in dementia care [2]. If and to what extent memory deficits can (temporarily) be reduced by communicating in an - positive or negative emotionally valent way, has, however, been studied less often. From clinical studies with somatically ill older people and with people without cognitive impairment, we know that emotions and memory go hand in hand; there is a positive association between information that carries an emotional valence and the extent to which this information is recalled correctly [3] ; [4], the so-called emotional memory advantage (EMA). In investigating this EMA, it is relevant to distinguish different types of emotional communication; emotions can be communicated by presenting emotional stimuli such as pictures, words, faces and stories, or by embedding (neutral) stimuli in an emotional context [5].

There are more than 50 different types of dementia associated with lesions in different parts of the brain. At present, 270,000 persons are affected with dementia in the Netherlands. Around seventy percent of them have Alzheimer's disease (AD), in some combined with another type of dementia, such as vascular dementia. In AD, patients show degeneration of the internal temporal regions (hippocampus and amygdala). The hippocampus is involved in episodic memory, the amygdala in memory of emotional messages [5]. In patients with other types of dementia, lesions in other regions may dominate impacting emotional processes in a different way. In 
Dulmen, S. van, Smits, L., Eide, H. Filling in memory gaps through emotional communication: promising pathways in caring for persons with dementia. Patient Education and Counseling: 2017, 100(11), 2121-2124

view of studying memory effects of emotional communication, it is also important to realize that the severity of dementia varies from mild to substantial cognitive impairments and can manifest itself in more or less maladaptive behavior and mood disturbances. So, information and emotions may be processed differently depending not only on someone's personality but also on the type and progression of the diagnosed dementia [6] ; [7]. The main aim of the paper is to explore the literature for empirical knowledge supporting the greater enhancement in recall by means of emotional stimuli compared to neutral stimuli in persons with dementia.

\section{METHODS}

As part of this rapid review [8] relevant studies published after 2000 were searched using Pubmed by applying different combinations of the terms: dementia, positive, words, communication, recall, and memory. A rapid review can be defined as "a type of knowledge synthesis in which components of the systematic review process are simplified or omitted to produce information in a short period of time" [8]. We aimed to include papers that reported results of studies with people with dementia that investigated memory effects of communication with either an emotionally valent content (e.g. pictures and words) or context (e.g. phrasing, tone or voice). The methods and results sections of the included studies were examined and described in chronological order.

\section{RESULTS}

All papers resulting from our search focused on investigating memory effects of presenting stimuli such as pictures, faces, words and stories in an experimental setting instead of a clinical setting. In this paper we describe the methods and results of the twelve papers grouped under four prevailing themes (pictures, facial emotions, stories and words). Whether the emotional stimuli were introduced in a more or less positive or neutral way has not been investigated at all.

\subsection{Pictures}

Kensinger and colleagues investigated memory for emotional versus neutral stimuli (pictures and words) in 20 young adults, 20 older adults, and thirteen patients with $\mathrm{AD}$ [9]. The AD patients did not show better memory for emotional compared with neutral items. Older adults and AD patients showed no benefit from the context of emotional words, whereas young adults did remember more items embedded in an emotional versus a neutral context. Perrin et al. studied the effect of the emotional valence of neutral, negative or positive pictures (animals, persons or scenes) preceded or followed by an emotional dialogue (context) on free and cued (immediate) recall in fifteen patients with mild $\mathrm{AD}$ and fifteen age-matched controls [5]. They showed that emotional content was related to recall in all subjects positive pictures being remembered better than neutral pictures - but positive context enhanced free recall of pictures in healthy elderly only. The positive context was drawn from movies, not from a subject's personal life. A few years later, GorencMahmutaj et al. investigated recognition and free recall of similar pictures in subjects with mild $(n=27)$ and moderate (Alzheimer's or vascular) dementia $(n=32)$, mild cognitive impairment $(n=32)$ and controls $(n=28)$ [4]. Overall, positive pictures were experienced with greater intensity. Positive pictures were also recalled more than negative pictures, both when assessed immediately and after $30 \mathrm{~min}$ (delayed 
Dulmen, S. van, Smits, L., Eide, H. Filling in memory gaps through emotional communication: promising pathways in caring for persons with dementia. Patient Education and Counseling: 2017, 100(11), 2121-2124

recall). Recognition was slightly better for positive pictures. Sundstrøm investigated free, immediate recall and recognition of emotional objects (associated with birthday gifts) in 20 patients with AD and 20 healthy controls [10]. He found that patients with AD recalled emotional objects significantly better than neutral objects (nongifts). No such emotional enhancement effect was found in the control group or when assessing recognition.

\subsection{Facial emotions}

By investigating the recognition of positive and negative facial emotions among 71 patients with $\mathrm{AD}$, Luzzi and colleagues found that this ability was largely preserved [11]. In $27 \%$ of the people, recognition was impaired. Maki et al. investigated the recognition of facial expressions (happiness, sadness, surprise, anger, disgust, and fear) and found that in patients with mild $\mathrm{AD}(\mathrm{n}=12)$, recognition of happiness was relatively preserved, also against the influence of age and disease [12].

\subsection{STORIES}

Kazui et al. investigated the effects of two short stories accompanying pictures in persons with $\mathrm{AD}(\mathrm{n}=34)$ and age-, gender- and education-matched healthy controls $(\mathrm{n}=10)$ [13]. In one story one passage was emotionally charged, in the other this passage was neutral. Both groups scored higher on recall of the story with emotionally charged content. Boller et al. examined whether the emotional content of a text displaying a neutral, happy or sad content influenced memory in ten patients with $\mathrm{AD}$ and twelve age-matched normal subjects [14]. They found that patients with $\mathrm{AD}$ recalled sad and, to a lesser extent, happy stories better than neutral stories. Similar findings resulted from assessing multiple choice recognition and identification of emotional content.

\subsection{Words}

Fleming and colleagues investigated immediate free recall of positive, negative and neutral emotionally valent words (e.g. friend, hate and thermometer, respectively) in patients with mild to moderate $\mathrm{AD}(\mathrm{N}=25)$ versus young $(\mathrm{n}=27)$ and old $(\mathrm{n}=19)$ controls [15]. AD patients appeared to recall more emotionally valent than neutral words. Besides, negative words were recalled more than positive or neutral words, especially in subjects with AD. Nieuwenhuis-Mark et al. also showed that, in patients with no $(n=38)$, mild $(n=37)$ or severe cognitive impairment/AD $(n=20)$, emotionally valent words are recalled better than neutral words (e.g. sky, bottle, poem) [16]. No evidence was found for a difference in effect between positive (e.g. Spring, angel, baby, beach) and negative (e.g. garbage, fire, poison, gun) words. In 2011, Billino et al. reported results of their study about the performance on an emotional word list learning task of fourteen patients with subcortical ischemic vascular dementia (SIVD), a diagnosis supported by emotional incontinence and lability [17]. Patients' performance was compared with that of healthy young control persons $(n=14)$ and old control persons $(n=14)$. The word list consisted of five neutral words (e.g. hall, month) and five negative words (e.g. betrayal, misery). Overall, subjects recalled more negative words than neutral words. SIVD patients consistently showed lower memory performance than the other groups in free recall as well as in recognition tasks. Yet, they did not appear to profit less from emotional information than the healthy controls. Kalenzaga et al. investigated memory of emotional versus neutral words (nouns) in eighteen AD patients and fifteen normal 
Dulmen, S. van, Smits, L., Eide, H. Filling in memory gaps through emotional communication: promising pathways in caring for persons with dementia. Patient Education and Counseling: 2017, 100(11), 2121-2124

controls [18]. They found that AD patients' memory was better for emotional compared to neutral words to the same extent as that of normal controls.

\subsection{Brief methodological reflection on the included studies}

The designs of the studies described varied considerably; some studies used controls, some focussed on different degrees of dementia and some included a single group exploration only. Few studies compared persons with dementia with healthy controls.

\section{DISCUSSION AND CONCLUSION}

\subsection{Discussion}

To sum up, the comparison in outcomes of persons with dementia and healthy controls tells us that, like normal controls, persons with dementia recall emotional stimuli better than neutral ones. Ten out of twelve studies described show that emotional stimuli increase recall compared with non-emotional stimuli among people with dementia. There is insufficient evidence that the severity of dementia is important.

In view of enhancing the memory of people with dementia, do we have to mind our words or wording? This (non-exhaustive) rapid review gives some indications in what way emotional communication influences short-term memory of people with dementia. Apparently, emotionally valent communication bears relevance for people's memory performance. Whether the effects of positive communication outweigh those of negative communication or vice versa is not yet convincing; not all studies examined effects of positive and negative stimuli, some compared negative with neutral stimuli only. Furthermore, in most studies arousal levels evoked by the stimuli were not controlled for. Besides, maybe for some people positive communication enhances memory, while for others, negative communication is more helpful. More research is needed in this area to find out how this function in a caring situation. We started our search looking for studies investigating positive emotions and therefore may have overlooked studies focusing on negative emotions only. So far, it is also unknown how long the memory enhancement created by emotion lasts. For the time being, caregivers do need to realize that their communication does have an influence and are advised to be especially alert on the use of emotions in their communication with the people with dementia and observe people's verbal and nonverbal responses.

The studies described test the effects of experimental interventions (words, pictures, faces) that may have low personal relevance for an individual and do not take the (momentarily) emotional valence of the communicated content for a particular person into account. The case example presented above focuses on spontaneously recalling a positive message that is different from the recognition or recall tests performed in the studies described. After all, from the 'generation effect' we know that self-generated information is remembered best [19], even more so when set in an emotionally valent context. As such implications for clinical practice may be limited. What is more, the persons performing the studies that are described in the included papers have no known personal relationship with the older person under study, which may diminish the emotional valence of the information they provided and diminish the clinical relevance of the findings. Besides, most studies are performed on people with (mild to moderate) $\mathrm{AD}$, the results of which are not generalizable to other types of dementia that affect other regions of the brain; dementia does not only affect a 
Dulmen, S. van, Smits, L., Eide, H. Filling in memory gaps through emotional communication: promising pathways in caring for persons with dementia. Patient Education and Counseling: 2017, 100(11), 2121-2124

person's memory. The studies described also included rather low numbers of subjects.

Emotional communication interventions could easily be designed, for example by focussing on providing positive feedback and using emotionally toned words and phrases while interacting with people with dementia [16]. When emotional communication indeed matters, caregivers can be taught to use it more purposefully in their contacts with patients.

This paper did not intend to find evidence for EMA and the positivity effect, merely to find input for the relevance of communicating in an emotionally valent way. On the basis of this rapid review, we cannot conclude if there is enough evidence to warrant a valence effect. To answer that question, a systematic review is needed. This rapid review does make clear that such a systematic review might be feasible and productive.

\subsection{Conclusion}

Emotional communication, either negative or positive, seems to enhance memory in people with dementia but how to use this knowledge in daily clinical care, is not clear yet. After all, none of the studies described focused on the association between personally relevant, emotionally valent information, such as information about a granddaughter's dream apartment, and memory. All studies have been done using test material like word lists, cards showing happy or sad faces, and the like. Direct observations and content analyses of interactions between people with dementia and their caregivers are needed to investigate the emotional memory advantage in everyday life.

\subsection{Practice implications}

Although we so far found mixed effects for the role of negative and positive emotionally valent communication, the results of this rapid review do make clear that in their contacts with people with dementia caregivers should realize that 1) their information might carry an emotional valence, and 2) this valence might influence the extent to which people remember information.

\section{Role of funding and conflict of interest}

No funding source(s) had any involvement in the preparation of this article.

\section{Conflict of interest}

No conflict of interest.

\section{Acknowledgements}

None.

\section{REFERENCES}

[1] M. Mather, L.L. Carstensen

Aging and motivated cognition: the positivity effect in attention and memory

Trends in Cognitivesciences (9) (2005), pp. 496-502

[2] J.C. van Weert, A.M. van Dulmen, P.M. Spreeuwenberg, M.W. Ribbe, J.M. Bensing

Behavioral and mood effects of snoezelen integrated into 24-hour dementia care

J. Am. Geriatr. Soc., 2005 (53) (2016), pp. 24-33

[3] J. Jansen, J. van Weert, J. de Groot, S. van Dulmen, T. Heeren, J. Bensing

Emotional and informational patient cues: the impact of nurses' responses on recall

Patient Educ. Couns., 79 (2010), pp. 218-224

[4] L. Gorenc-Mahmutaj, C. Degen, P. Wetzel, N. Urbanowitsch, J. Funke, J. Schröder 
Dulmen, S. van, Smits, L., Eide, H. Filling in memory gaps through emotional communication: promising pathways in caring for persons with dementia. Patient Education and Counseling:

2017, 100(11), 2121-2124

The positivity effect on the intensity of experienced emotion and memory performance in mild cognitive impairment and dementia

Dement. Geriatr. Cogn. Dis. Extra, 2015 (5) (2016), pp. 233-243

[5] M. Perrin, M.A. Henaff, C. Padovan, I. Faillenot, A. Merville, P. Krolak-Salmon

Influence of emotional content and context on memory in mild Alzheimer's disease

J. Alzheimers. Dis., 2012 (29) (2016), pp. 817-826

[6] I. Rouleau, H. Imbault, M. Laframboise, M.A. Bédard

Pattern of intrusions in verbal recall: comparison of Alzheimer's disease, Parkinson's

disease, and frontal lobe dementia

Brain Cogn., 46 (2001), pp. 244-249

[7] K. Fleming, S.H. Kim, M. Doo, G. Maguire, S.G. Potkin

Memory for emotional stimuli in patients with Alzheimer's disease

Am. J. Alzheimers Dis. Other. Demen., 18 (2003), pp. 340-342

[8] A.C. Tricco, J. Antony, W. Zarin, L. Strifler, M. Ghassemi, J. Ivory, L. Perrier, B. Hutton, D. Moher, S.E. Straus

A scoping review of rapid review methods

BMC. Med., 2015 (13) (2016), p. 224

[9] E.A. Kensinger, B. Brierley, N. Medford, J.H. Growdon, S. Corkin

Effects of normal aging and Alzheimer's disease on emotional memory

Emotion, 2002 (2) (2016), pp. 118-134

[10] M. Sundstrøm

Modeling recall memory for emotional objects in Alzheimer's disease

Aging Neuropsychol. Cogn., 2011 (18) (2016), pp. 396-413

[11] S. Luzzi, M. Piccirilli, L. Provinciali

Perception of emotions on happy/sad chimeric faces in Alzheimer disease: relationship with cognitive functions

Alzheimer Dis. Assoc. Disord. (21) (2007), pp. 130-135

[12] Y. Maki, H. Yoshida, T. Yamaguchi, H. Yamaguchi

Relative preservation of the recognition of positive facial expression 'happiness' in Alzheimer disease

Int. Psychogeriatr., 25 (2013), pp. 105-110

[13] H. Kazui, E. Mori, M. Hashimoto, N. Hirono, T. Imamura, S. Tanimukai, T. Hanihara, L. Cahill

Impact of emotion on memory. Controlled study of the influence of emotionally charged material on declarative memory in Alzheimer's disease

Br. J. Psychiatry, 2000 (177) (2016), pp. 343-347

[14] F. Boller, F. El Massioui, E. Devouche, L. Traykov, S. Pomati, S.E. Starkstein

Processing emotional information in Alzheimer's disease: effects on memory performance and neurophysiological correlates

Dement. Geriatr. Cogn. Disord., 2002 (2) (2016), pp. 104-112

[15] K. Fleming, S.H. Kim, M. Doo, G. Maguire, S.G. Potkin

Memory for emotional stimuli in patients with Alzheimer's disease

Am. J. Alzheimer's Dis. Other Dement., 18 (2003), pp. 340-342

[16] R.E. Nieuwenhuis-Mark, K. Schalk, N. de Graaf

Free recall and learning of emotional word lists in very elderly people with and without dementia

Am. J. Alzheimers Dis. Other Demen., 24 (2009), pp. 155-162

[17] J. Billino, J. Luerssen, W. von Renteln-Kruse, C. Mühlhan, A. Rösler

Effects of subcortical vascular ischemic dementia and aging on negative and neutral word list learning

Dement. Geriatr. Cogn. Disord., 31 (2011), pp. 188-194

[18] S. Kalenzaga, P. Piolino, D. Clarys

The emotional memory effect in Alzheimer's disease: emotional words enhance recollective experience similarly in patients and control participants

Cogn. Emot., 29 (2015), pp. 342-350

[19] N.J. Slamecka, P. Graf

The generation effect: delineation of a phenomenon

J. Exp. Psychol. Hum. Learn. Memory, 1978 (4) (2016), pp. 592-604 